\title{
Comparison of Three Molecular Assays for the Detection of Rifampin Resistance in Mycobacterium tuberculosis
}

\author{
Hee-Won Moon, Mina Hur, ${ }^{*} \mathrm{Ji}-$ Young Kim, and Yeo-Min Yun \\ Department of Laboratory Medicine, Konkuk University School of Medicine, Konkuk University Hospital, \\ Seoul, Korea
}

Background: Rifampin (RIF) is the most important first-line antituberculosis drug, and resistance to this drug may result in treatment failures. We evaluated the diagnostic performances of recently introduced, molecular assays for the detection of RIF resistance. Methods: A total of 100 isolates (50 RIF resistant and 50 RIF susceptible) were studied. Their RIF resistances were determined by conventional drug-susceptibility test. These results were compared with those of three molecular assays: Xpert MTB/RIF assay (MTB is Mycobacterium tuberculosis), Sacace MTB Real-TM resistance, and AdvanSure MDR-
TB GenoBlot assay (MDR is multidrug resisitant). Results: Sensitivities for RIF resistance detection of Xpert MTB/RIF assay, Sacace MTB Real-TM resistance, and Advansure GenoBlot assay were $94.0 \%$, $91.8 \%$, and $84.0 \%$, respectively. Their specificities for RIF resistance detection were all $100 \%$. Conclusion: Three molecular assays for the detection of RIF resistance have various performances. Xpert MTB/RIF assay shows the highest sensitivity among the three molecular assays and can be an effective choice in clinical laboratories. J. Clin. Lab. Anal. 29:142-145, 2015.

2014 Wiley Periodicals, Inc

Key words: tuberculosis; resistance; rifampin; molecular assay

\section{INTRODUCTION}

The burden of tuberculosis (TB) remains enormous worldwide. The mortality rate of TB is still high, and there were 1.7 million human deaths due to TB in 2009 (1). Rifampin (RIF) is the most important first-line anti-TB drug, and resistance to this drug may result in treatment failures. Globally, the emergence and spread of multidrugresistant (MDR) and extensively drug-resistant (XDR) TB are increasing concerns and cause significant challenges to control this disease (2). A recent report revealed that $20 \%$ of the isolates met the MDR criteria and $2 \%$ of them were classified as XDR (3).

Although culture-based, conventional drug-sensitivity testing (DST) has been considered gold standard to detect drug resistance of Mycobacterium tuberculosis (MTB), it is time consuming and labor intensive, causing a diagnostic delay. To prevent and control the spread of MDR and XDR TB, a simple and convenient DST is essential.

Recently, the Xpert MTB/RIF assay (Cepheid, Sunnyvale, $\mathrm{CA}$ ) was introduced and was endorsed by World Health Organization (WHO) (4). This assay simultane- ously detects the presence of MTB and its susceptibility to RIF in a single reaction, which integrates sample processing and polymerase chain reaction in a disposable plastic cartridge (5-7). Because most RIF-resistant isolates also exhibit resistance to isoniazid (INH), the detection of RIF resistance serves as a surrogate marker for MDR MTB (8). Also, several molecular assays for the detection of RIF resistance have been recently developed. In this study, we wanted to evaluate the diagnostic performances of recently introduced, molecular assays for the detection of RIF resistance.

Grant sponsor: This work was supported by Konkuk University Medical Center Research Grant 2013.

*Correspondence to: Mina Hur, Department of Laboratory Medicine, Konkuk University School of Medicine, Konkuk University Hospital, 120-1, Neungdong-ro, Hwayang-dong, Gwangjin-gu, Seoul 143-729, Korea. E-mail: dearmina@hanmail.net

Received 10 December 2012; Accepted 8 January 2014

DOI 10.1002/jcla. 21742

Published online in Wiley Online Library (wileyonlinelibrary.com). 


\section{MATERIALS AND METHODS}

\section{Clinical Isolates}

A total of 100 , nonduplicated isolates (50 RIF resistant and 50 RIF susceptible) from respiratory specimens of the patients with TB were studied. Their RIF resistance was determined by conventional DST (resistance ratio method). The results of DST were considered gold standard and were compared with those of three molecular assays: Xpert MTB/RIF assay, Sacace MTB RealTM resistance (Sacace Biotechnologies, Como, Italy), and AdvanSure MDR-TB GenoBlot assay (LG Life Sciences, Seoul, Korea).

\section{Xpert MTB/RIF Assay}

The Xpert MTB/RIF assay is an automated assay in sample processing, nucleic acid amplification, and detection of the target sequences in simple or complex samples using real-time PCR. The system requires the use of single-use disposable Xpert cartridges that hold the PCR reagents and host the PCR process. The primers in the Xpert MTB/RIF assay amplify a portion of the $r p o B$ gene containing the 81-bp "core" region. The probes are able to differentiate between the conserved wild-type sequence and mutations in the core region that are associated with RIF resistance.

\section{Sacace MTB Real-TM Resistance}

MTB Real-TM resistance is an in vitro allele-specific real-time PCR test for specific mutations in rpoB, katG, and inh $A$ genes associated with INH or RIF resistance: rpo $B$ codon 531 (two mutations: Ser-Leu and Ser-Trp), rpoB codon 526-1 (three mutations: His-Tyr, His-Asp, and His-Arg), rpoB codon 526-2 (three mutations: HisLeu, His-Asn, and His-Pro), rpoB codon 516 (one mutation: Asp-Val), rpoB codon 533 (one mutation: Leu-Pro), and $k a t G$ codon 315 (three mutations: Ser-Thr, Ser-Asn, and Ser-Thr).

\section{AdvanSure MDR-TB GenoBlot Assay}

AdvanSure MDR-TB GenoBlot assay is a reversehybridization line blot assay using one-tube nested multiplex asymmetric PCR, targeting rpoB, katG, inh $A$, and $a h p C$ genes. This assay has 21 strip lines, which include 14 for RIF resistance, 2 for INH high-level resistance, and 5 for INH low-level resistance.

\section{Statistical Analysis}

Sensitivities and specificities for each assay were calculated with 95\% confidence interval (CI). The McNemar's test was used to decide the statistical difference between assays. Statistical analysis was performed using SPSS software, (version 12.0, SPSS Inc., Chicago, IL), MedCalc Statistical Software (version 11.2.1, MedCalc Software, Mariakerke, Belgium), and Analyse-it Method Evaluation Edition (Analyse-it Software, Ltd., Leeds, UK). $P$-values less than 0.05 were considered statistically significant.

\section{RESULTS}

Among the 50 RIF-resistant isolates, Xpert MTB/RIF assay detected the RIF resistance in 47 isolates, Sacace MTB Real-TM resistance in 45 isolates, and Advansure GenoBlot assay in 42 isolates (Table 1). False resistance was not detected in three assays with 50 RIF-susceptible isolates.

Sensitivities for RIF resistance detection of Xpert MTB/RIF assay, Sacace MTB Real-TM resistance, and Advansure GenoBlot assay were 94.0\%, 91.8\%, and $84.0 \%$, respectively. Their specificities for RIF resistance detection were all $100 \%$. Compared to culture-based DST, the results of Xpert MTB/RIF assay and MTB Real-TM resistance were not different, but Advansure GenoBlot assay showed a significant difference $(P=0.0078)$.

\section{DISCUSSION}

The Xpert MTB/RIF assay is a WHO-endorsed diagnostic tool and has many advantages, including simultaneous detection of MTB and RIF resistance, automated processing, reaction in disposable plastic cartridge, and rapid turnaround time (4-6). Recent studies have evaluated the performance of Xpert MTB/RIF assay for MTB detection, and the reported sensitivities were about 98.0 $100.0 \%$ in smear-positive, culture-positive patients and $57.0-72.5 \%$ in smear-negative, culture-positive patients (5,9-11). Recent reports also evaluated the performances of Xpert MTB/RIF assay for MTB detection in nonrespiratory specimens or in HIV-positive patients, and the results of these studies were variable and need to be confirmed through further studies $(10,12-14)$.

To the best of our knowledge, the studies on the performance of Xpert MTB/RIF assay for detection of RIF resistance are sparse (13), and most studies included only limited number of resistant isolates $(14,15)$. Moreover, the performances of MTB Real-TM resistance and AdvanSure MDR-TB GenoBlot assay have not been evaluated so far. This study has focused on the performance of detection of RIF resistance and compared the results of three molecular assays for resistance of RIF.

The Xpert MTB/RIF assay showed excellent sensitivity $(94.0 \%)$ and specificity $(100.0 \%)$ for RIF resistance. A 
TABLE 1. Performances of Three Molecular Assays for Detection of RIF Resistance in Comparison to Culture-Based Method

\begin{tabular}{lcccccccc}
\hline Test method & $\begin{array}{c}\text { No. of } \\
\text { samples }\end{array}$ & $\begin{array}{c}\text { True } \\
\text { positive }\end{array}$ & $\begin{array}{c}\text { False } \\
\text { positive }\end{array}$ & $\begin{array}{c}\text { True } \\
\text { negative }\end{array}$ & $\begin{array}{c}\text { False } \\
\text { negative }\end{array}$ & Sensitivity (95\% CI) & Specificity (95\% CI) & $P^{\text {a }}$ \\
\hline Xpert MTB/RIF & 100 & 47 & 0 & 50 & 3 & $94.0 \%(83.5-98.8)$ & $100.0 \%(92.9-100.0)$ & NS \\
Sacace MTB Real-TM resistance & 100 & 45 & 0 & 50 & 5 & $91.8 \%(80.4-97.7)$ & $100.0 \%(92.9-100.0)$ & NS \\
AdvanSure MDR-TB GenoBlot & 100 & 42 & 0 & 50 & 8 & $84.0 \%(70.9-92.8)$ & $100.00 \%(92.9-100.0)$ & 0.0078 \\
\hline
\end{tabular}

${ }^{a}$ There was no statistical difference between the results for any two molecular assays $(P>0.05$, McNemar's test).

recent study also showed similar performance of Xpert MTB/RIF assay (sensitivity $94.4 \%$ and specificity $98.3 \%$ for RIF resistance) (13). On the other hand, Salvo et al. (16) concerned the discrepant results between results of the Xpert MTB/RIF test for RIF resistance and those of conventional DST. They suggested that the results of Xpert MTB/RIF test need to be confirmed with a second test or conventional DST, before treatment for MDR TB.

MTB Real-TM resistance also showed excellent sensitivity and specificity (sensitivity $91.8 \%$ and specificity $100.0 \%$ ) for RIF resistance, but its sensitivity was lower compared with that of Xpert MTB/RIF assay. AdvanSure MDR-TB GenoBlot assay showed lower sensitivity $(84.0 \%)$ for RIF resistance compared with the other assays. Principle of AdvanSure MDR-TB GenoBlot assay is different from the other two assays. Blot hybridization does not require real-time PCR device, therefore it could be more optimal to the small-setting laboratory. Since we did not perform DNA sequencing and the results were compared with those of conventional DST, the reasons of false-negative results could be related to low sensitivity of assay to detect mutations or the resistance due to mechanisms other than covered mutations.

Overall, Xpert MTB/RIF assay and MTB Real-TM resistance showed excellent performance for detection of RIF resistance. The Xpert MTB/RIF assay is fast, technically simple and can be performed as a point-of-care test, while MTB Real-TM resistance and AdvanSure MDRTB GenoBlot assay require the more complex processing step and separate DNA extraction step, which take more hands-on time and manual workload. Moreover, the result of AdvanSure MDR-TB GenoBlot assay needs to be further evaluated using more specimens with various susceptibilities. In conclusion, our data show that molecular assays for the detection of RIF resistance have various performances. The Xpert MTB/RIF assay shows the highest sensitivity among the three molecular assays and can be an effective choice in routine clinical laboratories.

\section{ABBREVIATIONS}

$\mathrm{CI}=$ confidence interval

DST $=$ drug sensitivity testing
$\mathrm{MDR}=$ multidrug resistant

MTB = Mycobacterium tuberculosis

NS $=$ not significant

RIF $=$ Rifampin

$\mathrm{TB}=$ tuberculosis

$\mathrm{XDR}=$ extensively drug-resistant

\section{REFERENCES}

1. WHO. 2010/2011 Tuberculosis Global Facts, Geneva: WHO; 2011. Available from: http://www.who.int/tb/publications/2010/ factsheet_tb_2010.pdf.

2. WHO. Multidrug and Extensively Drug-Resistant TB (M/XDRTB): 2010 Global Report on Surveillance and Response, WHO/HTM/TB/2010.3, Geneva: WHO; 2010.

3. Centers for Disease Control and Prevention (CDC). Emergence of Mycobacterium tuberculosis with extensive resistance to secondline drugs-worldwide, 2000-2004. MMWR Morb Mortal Wkly Rep 2006;55:301-305.

4. WHO. WHO endorses new rapid tuberculosis test. 2010. Available from: http://www.who.int/mediacentre/news/releases/ 2010/tb_test_20101208/en/index.html. Accessed 15 June 2011.

5. Boehme CC, Nabeta P, Hillemann D, et al. Rapid molecular detection of tuberculosis and rifampin resistance. N Engl J Med 2010;363:1005-1015.

6. Helb D, Jones M, Story E, et al. Rapid detection of $M y$ cobacterium tuberculosis and rifampin resistance by use of ondemand, near-patient technology. J Clin Microbiol 2010;48: 229-237.

7. Blakemore R, Story E, Helb D, et al. Evaluation of the analytical performance of the Xpert MTB/RIF assay. J Clin Microbiol 2010;48:2495-2501.

8. Watterson SA, Wilson SM, Yates MD, Drobniewski FA. Comparison of three molecular assays for rapid detection of rifampin resistance in Mycobacterium tuberculosis. J Clin Microbiol 1998;36:1969-1973.

9. Marlowe EM, Novak-Weekley SM, Cumpio J, et al. Evaluation of the Cepheid Xpert MTB/RIF assay for direct detection of $M y$ cobacterium tuberculosis complex in respiratory specimens. J Clin Microbiol 2011;49:1621-1623.

10. Armand S, Vanhuls P, Delcroix G, Courcol R, Lemaitre N. Comparison of the Xpert MTB/RIF test with an IS6110-TaqMan realtime PCR assay for direct detection of Mycobacterium tuberculosis in respiratory and nonrespiratory specimens. J Clin Microbiol 2011;49:1772-1776.

11. Teo J, Jureen R, Chiang D, Chan D, Lin R. Comparison of two nucleic acid amplification assays, the Xpert MTB/RIF and the Amplified Mycobacterium Tuberculosis Direct (MTD) assay, for the detection of Mycobacterium tuberculosis in respiratory and non-respiratory specimens. J Clin Microbiol 2011;49: 3659-3662. 
12. Theron G, Peter J, van Zyl-Smit R, et al. Evaluation of the Xpert MTB/RIF assay for the diagnosis of pulmonary tuberculosis in a high HIV prevalence setting. Am J Respir Crit Care Med 2011;184:132-140.

13. Boehme CC, Nicol MP, Nabeta P, et al. Feasibility, diagnostic accuracy, and effectiveness of decentralised use of the Xpert MTB/RIF test for diagnosis of tuberculosis and multidrug resistance: A multicentre implementation study. Lancet 2011;377:1495-1505.

14. Scott LE, McCarthy K, Gous N, et al. Comparison of Xpert MTB/RIF with other nucleic acid technologies for diagnosing pulmonary tuberculosis in a high HIV prevalence setting: A prospective Study. PLoS Med 2011;8:e1001061.

15. Ioannidis P, Papaventsis D, Karabela S, et al. Cepheid Xpert MTB/RIF assay for Mycobacterium tuberculosis detection and rifampin resistance identification in patients with substantial clinical indications of tuberculosis and smear-negative microscopy results. J Clin Microbiol 2011;49:3068-3070.

16. Salvo F, Sadutshang TD, Migliori GB, Zumla A, Cirillo DM. Xpert MTB/RIF test for tuberculosis. Lancet 2011;378:481-482; author reply $2-3$. 